JOURNAL OF SYNCHROTRON RADIATION

ISSN 1600-5775

Received 16 January 2020

Accepted 30 November 2020

Edited by V. Favre-Nicolin, ESRF and Université Grenoble, France

₹ These authors contributed equally to this study.

Keywords: partial coherence; wave optics model; coherent diffractive imaging; high-energy photon sources; Young's double-slits interference.
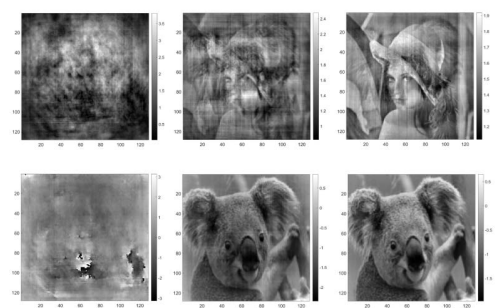

(C) 2021 International Union of Crystallography

\section{A wave optics model for the effect of partial coherence on coherent diffractive imaging}

\author{
Zhongzhu Zhu,‡ Han Xu,‡ Lingfei Hu, Ming Li, Peng Liu, Yuhui Dong and \\ Liang Zhou*
}

Beijing Synchrotron Radiation Facility, Institute of High Energy Physics, Chinese Academy of Sciences, Beijing, People's Republic of China. *Correspondence e-mail: zhouliang@ihep.ac.cn

With the development of fourth-generation synchrotron sources, coherent diffractive imaging (CDI) will be a mainstream method for 3D structure determination at nanometre resolution. The partial coherence of incident $\mathrm{X}$-rays plays a critical role in the reconstructed image quality. Here a wave optics model is proposed to analyze the effect of partial coherence on CDI for an actual beamline layout, based on the finite size of the source and the influence of the optics on the wavefront. Based on this model, the light field distribution at any plane, the coherence between any two points on this plane and CDI experiments can be simulated. The plane-wave CDI simulation result also shows that in order to reconstruct good image quality of complex samples the visibility of the interference fringes of any two points in the horizontal and vertical directions of the incident light field at the sample needs to be higher than 0.95 .

\section{Introduction}

Coherent X-ray diffraction imaging (CDI) is a coherent scattering technique where the far-field diffraction intensity is used to reconstruct the $2 \mathrm{D}$ or $3 \mathrm{D}$ structure of samples by phase iterative algorithm (Miao et al., 2015; Chapman \& Nugent, 2010). Since the first experimental demonstration by Miao et al. (1999), CDI has been used to obtain structures of nanocrystalline and polycrystalline materials (Pfeifer et al., 2006; Ulvestad et al., 2015; Yau et al., 2017), non-crystalline specimens (Miao et al., 2006; Jiang et al., 2013; Song et al., 2014; Kim et al., 2017) and extended samples (Dierolf et al., 2010; Diaz et al., 2015; Holler et al., 2017) with X-ray synchrotron radiation or X-ray free-electron lasers (XFELs). The resolution for inorganic samples is about $10 \mathrm{~nm}$ (Miao et al., 2015), and that for biological samples is about tens of nanometres (Song et al., 2014; Kim et al., 2017). Therefore, there is still room for improvement of the resolution. The resolution $d$ mainly depends on the maximum scattering angle $\vartheta$ by Bragg's law: $2 d \sin \vartheta=\lambda$, where $\lambda$ is the wavelength. With increasing scattering angle the diffraction intensity $I$ (i.e. photons per pixel) and signal-to-noise ratio (SNR) decrease. Thus, the resolution is limited by the SNR; for example, $I>5$, SNR = $I / \sigma_{I}>2$ (Huang et al., 2009; Shen et al., 2004; Neutze et al., 2000). With the construction of fourth-generation synchrotron radiation sources, the coherent flux will be increased by at least two orders (Advanced Photon Source, 2017; ESRF, 2015), and the signal-to-noise ratio can be improved by one order (Shen et al., 2004), which could highly increase the resolution of CDI.

The reconstruction quality of CDI depends on the contrast of the diffraction pattern, which is affected by many factors. 
Partial coherence, for example, is one of the most important factors to be considered (Williams et al., 2007; Whitehead et al., 2009). At present, a commonly used method to consider the effect of partial coherence is via the mutual coherence function (MCF) or mutual optical intensity (MOI) (Nugent, 2010; Clark et al., 2012). Based on this theory, the intensity of the diffraction pattern in reciprocal space is the convolution of the Fourier transform of the normalized MCF and the fully coherent diffraction intensity, which is obtained through the usual Fourier transform of the exiting light field. In order to analyze the effect of partial coherence on CDI, it is necessary to calculate the MOI at the sample in the actual beamline. MOI analysis of the partial coherence on a single optical device has been performed (Meng et al., 2017). However, since the wave from the source will go through a series of optics with errors in order to reach the sample (determined by the optical layout), which strongly increases the amount of computation of the MOI convolution, MOI analysis of the partial coherence effect on CDI is complicated to perform.

In this paper, a wave propagation model is developed to analyze the partial coherence effect on CDI for an actual beamline, the Hard X-ray Coherent Scattering beamline of the High Energy Photon Source (HEPS), Beijing, China. In this model, the source is simulated as a composition of independent point sources, and the Fresnel-Kirchhoff integral is used to calculate the wave propagation. The spatial partial coherence of the light wave is controlled by the propagation distance from the secondary source to the sample. The visibility of double-slit interference is used to measure the partial coherence. By performing the CDI simulation under incident $\mathrm{X}$-rays with different partial coherence, the reconstruction quality of the diffraction pattern and the partial coherence are related.

\section{Model description and numerical analysis}

In general, a multi-electron Monte Carlo simulation, such as Synchrotron Radiation Workshop (SRW; Chubar \& Elleaume, 1998) or X-Ray Tracer (XRT; Klementiev \& Chernikov, 2014), is used to simulate the wave optics of a beamline. However, the huge amount of computation involved in the multi-electron Monte Carlo simulation is beyond the ability of most desktop computers. Thus, in this model, the source is simulated as the composition of independent point sources. The weight of each point source is determined by the intensity distribution of the source, which is Gaussian, and the phase of each point source is set randomly. The spherical wave produced by a single point source propagates through the optics in the beamline.

As shown in Fig. 1, the optical layout of the Hard X-ray Coherent Scattering beamline of HEPS is used in this wave optics model. The locations of optical devices have been labeled in the figure. A point source $s$ at the source plane generates a spherical wave that propagates to the incident plane of the first optics,

$$
U_{\mathrm{s}}^{\mathrm{i}}\left(x_{1}, y_{1}\right)=\sqrt{I\left(x_{0}, y_{0}\right)} \exp \left[j \varphi\left(x_{0}, y_{0}\right)\right] \frac{\exp (j k r)}{r},
$$

where $I\left(x_{0}, y_{0}\right)$ obeys a Gaussian distribution, $\varphi\left(x_{0}, y_{0}\right)$ is a random phase, $k$ is the wavenumber and $r$ is the distance between $\left(x_{0}, y_{0}\right)$ and $\left(x_{1}, y_{1}\right)$. The complex amplitude distribution at the output surface of the first optics is as follows,

$$
U_{\mathrm{s}}\left(x_{1}, y_{1}\right)=U_{\mathrm{s}}^{\mathrm{i}}\left(x_{1}, y_{1}\right) \exp \left[i M\left(x_{1}, y_{1}\right)\right],
$$

where $M\left(x_{1}, y_{1}\right)$ is the wavefront modulation function of the focusing optics (i.e. ideal lens in Fig. 1).

After passing through the first optics, the wavefront propagates to the downstream optics through the Kirchhoff diffraction integral,

$$
\begin{aligned}
U_{\mathrm{s}}\left(x_{n}, y_{n}\right)= & \frac{1}{j \lambda} \iint U_{\mathrm{s}}\left(x_{n-1}, y_{n-1}\right) \frac{\exp \left(j k r_{n-1, n}\right)}{r_{n-1, n}} \\
& \times \cos \left(\theta_{n-1, n}\right) \mathrm{d} x_{n-1} \mathrm{~d} y_{n-1}, \quad n \geq 2,
\end{aligned}
$$

where $r_{n-1, n}$ is the distance between $\left(x_{n-1}, y_{n-1}\right)$ and $\left(x_{n}, y_{n}\right)$, and $\theta_{n-1, n}$ is the angle between $\mathbf{r}_{n-1, n}$ and the optical axis.

The intensities of these propagated waves are summed up at the plane of interest (sample, for example) to generate the total intensity,

$$
I\left(x_{n}, y_{n}\right)=\sum_{s}\left|U_{\mathrm{s}}\left(x_{n}, y_{n}\right)\right|^{2} .
$$

Young's double-slit interference is used to measure the coherence of the light field. The double-slit was placed at different locations, with the screen $1 \mathrm{~m}$ behind them. By calculating the visibility of the interference at different locations with equations (3) and (4), the partial coherence is characterized. Then, the double-slit is replaced by a twodimensional complex sample to perform a simulation of the CDI experiment. For the CDI simulation, a fast Fourier transform (FFT) is used to calculate the diffraction pattern. The scattering intensity corresponding to a one-point source $s$ is 


$$
\begin{aligned}
I_{\mathrm{s}}\left(Q_{x}, Q_{y}\right)= & \left|U_{\mathrm{s}}\left(Q_{x}, Q_{y}\right)\right|^{2} \\
= & \mid \iint U_{\mathrm{s}}\left(x_{n}, y_{n}\right) T\left(x_{n}, y_{n}\right) \\
& \times\left.\exp \left[j\left(Q_{x} x_{n}+Q_{y} y_{n}\right)\right] \mathrm{d} x_{n} \mathrm{~d} y_{n}\right|^{2},
\end{aligned}
$$

where $Q_{x}$ and $Q_{y}$ are reciprocal space variables, $U_{\mathrm{s}}\left(x_{n}, y_{n}\right)$ is the complex amplitude distribution at the sample plane, and $T\left(x_{n}, y_{n}\right)$ is the transmission function of the sample, located at $z_{3}$ in our beamline layout. The total scattering intensity is generated by the summation of all scattering intensity produced by every weighted point source,

$$
I\left(Q_{x}, Q_{y}\right)=\sum I_{\mathrm{s}}\left(Q_{x}, Q_{y}\right)
$$

The above model can be used to simulate our beamline. The parameters of the light source are as follows. The energy of the source is $12.4 \mathrm{keV}$. The intensity distribution of the source is Gaussian with $\sigma_{\text {horizontal }}=9.5 \mu \mathrm{m}$ and $\sigma_{\text {vertical }}=3.1 \mu \mathrm{m}$. The following propagation is simulated by equations (1) to (6).

Assuming the focusing optics is an ideal lens, it can be easily expressed as a wavefront modulation function as appeared in equation (2),
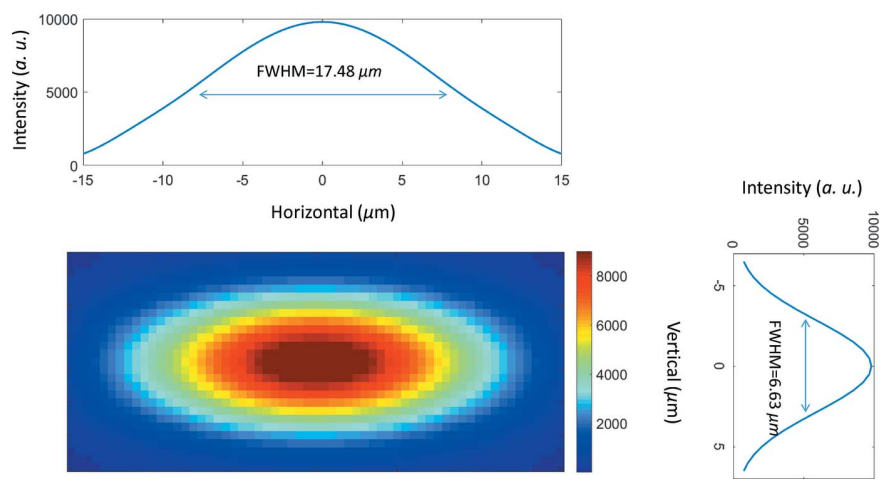

Figure 2

The secondary source and the profiles along the horizontal and vertical directions.
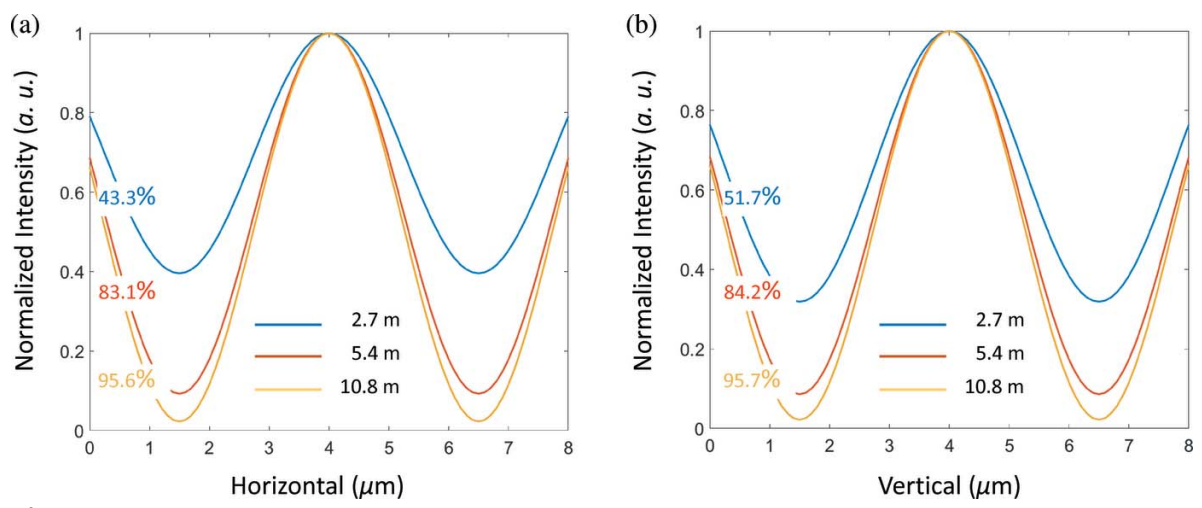

Figure 3

The $(a)$ horizontal and $(b)$ vertical double-slit interference results at $2.7 \mathrm{~m}, 5.4 \mathrm{~m}$ and $10.8 \mathrm{~m}$. The responding visibilities at different locations are labeled.
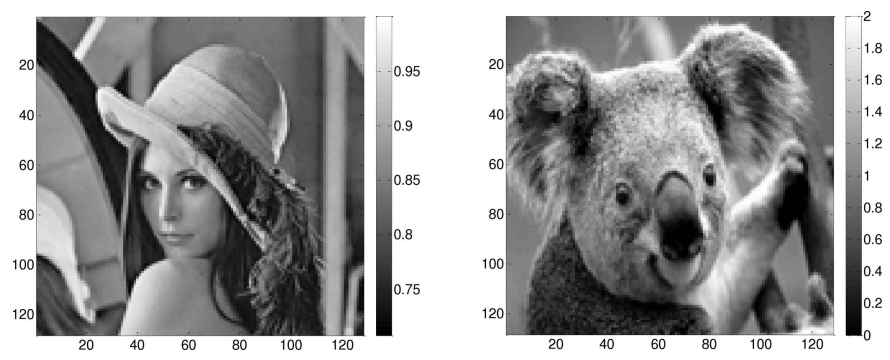

Figure 4

Standard Lena and Koala figures are used as the amplitude and phase, respectively, of the two-dimensional complex sample, with a size of $20 \mu \mathrm{m} \times 20 \mu \mathrm{m}(128 \times 128$ grids $)$.

$$
\begin{aligned}
M\left(x_{1}, y_{1}\right)= & \varphi_{\text {convergent }}\left(x_{1}, y_{1}, z_{1}, z_{2}\right)-\varphi_{\text {divergent }}\left(x_{1}, y_{1}, z_{1}\right) \\
= & -\frac{2 \pi}{\lambda}\left[x_{1}^{2}+y_{1}^{2}+\left(z_{2}-z_{1}\right)^{2}\right]^{1 / 2} \\
& -\frac{2 \pi}{\lambda}\left[x_{1}^{2}+y_{1}^{2}+z_{1}^{2}\right]^{1 / 2},
\end{aligned}
$$

where $\varphi_{\text {convergent }}\left(x_{1}, y_{1}, z_{1}, z_{2}\right)$ and $\varphi_{\text {divergent }}\left(x_{1}, y_{1}, z_{1}\right)$ are, respectively, the phase of the convergent spherical wave at the output surface of the focusing optics and the phase of the divergent spherical wave at the incident surface of the focusing optics, and $z_{1}$ and $z_{2}$ are the locations of the ideal lens and the focus spot (Fig. 1). In addition, it is convenient to add the error of the ideal lens in the following work. The secondary source (Fig. 2) is obtained by the compound refractive lens (ideal lens), the aperture of which is $600 \mu \mathrm{m}$. The vertical and horizontal FWHM is $6.6 \mu \mathrm{m}$ and $17.5 \mu \mathrm{m}$, respectively, which is consist with the theoretical value of $8.2 \mu \mathrm{m}$ and $17 \mu \mathrm{m}$. In order to make the horizontal and vertical coherence close to each other at the sample, the secondary source aperture (SSA) in the horizontal direction is set to $10 \mu \mathrm{m}$, with no SSA in the vertical direction.

Young's experiment is performed along both horizontal and vertical directions. The spacing of the double-slit is $20 \mu \mathrm{m}$. The double-slits are placed at $2.7 \mathrm{~m}, 5.4 \mathrm{~m}$ and $10.8 \mathrm{~m}$ after the secondary source; and the detector is placed $1.0 \mathrm{~m}$ after the double-slit with a pixel size of $0.1 \mu \mathrm{m} \times 0.1 \mu \mathrm{m}$. As shown in Fig. 3, the visibility of interference fringes increases with the distance from the double-slit to the secondary source. The visibility of the interference fringes is used to characterize the partial coherence ( 0 for incoherent, 1 for total coherence). Thus, the coherence decreases with decreasing distance and the effect of partial coherence increase, which reduces the visibility of the interference.

Then the double-slit at different locations is replaced by a two-dimensional complex sample. Thus, for samples at different locations, the partial coherence of the incident light 
(a)

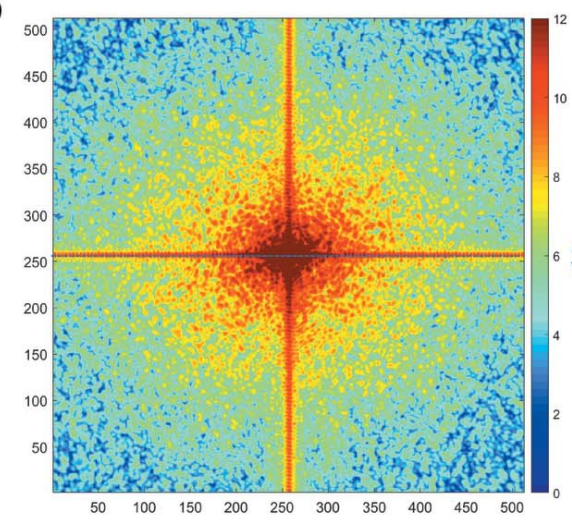

(c)

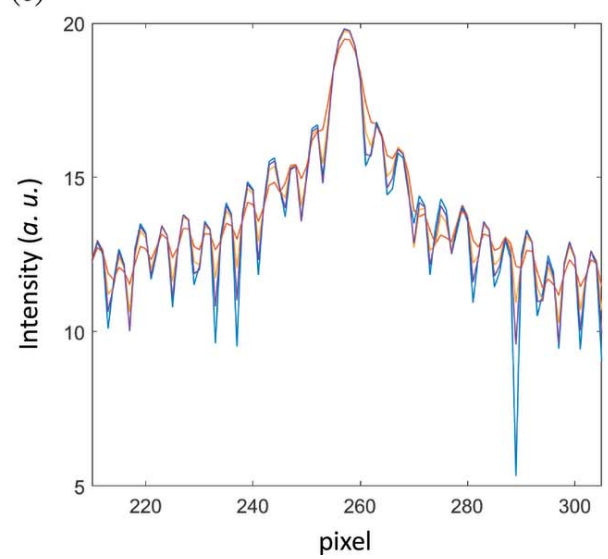

(b)

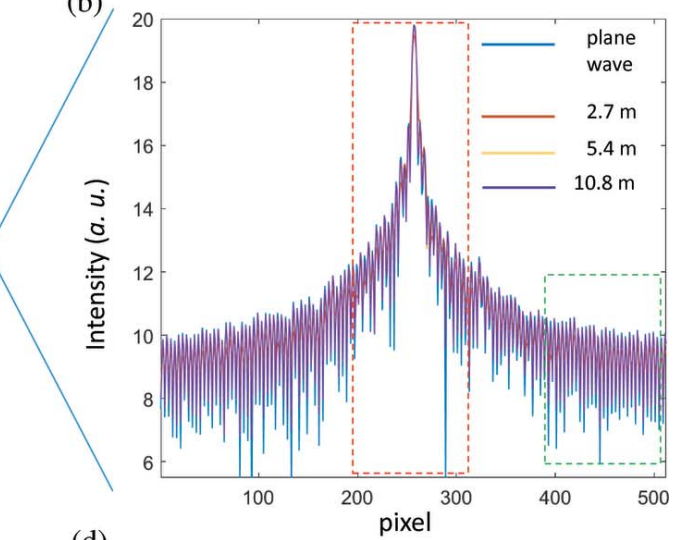

(d)

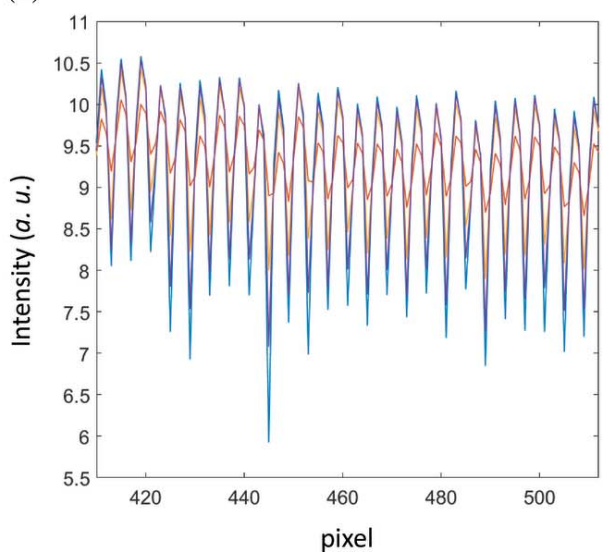

Figure 5

(a) The simulated diffraction pattern of the sample at $2.7 \mathrm{~m}$. (b) The profiles along the horizontal direction at $2.7 \mathrm{~m}, 5.4 \mathrm{~m}$ and $10.8 \mathrm{~m}$. The result of the plane wave corresponding to the full coherence case is also shown. The details of the diffraction pattern at $(c)$ high and $(d)$ low spatial frequency.

field is modulated by the distances. For CDI simulation, standard Lena and Koala figures are used as the amplitude and phase, respectively, of the sample shown in Fig. 4, the size of which is $20 \mu \mathrm{m} \times 20 \mu \mathrm{m}(128 \times 128$ grids $)$. The locations of (a)
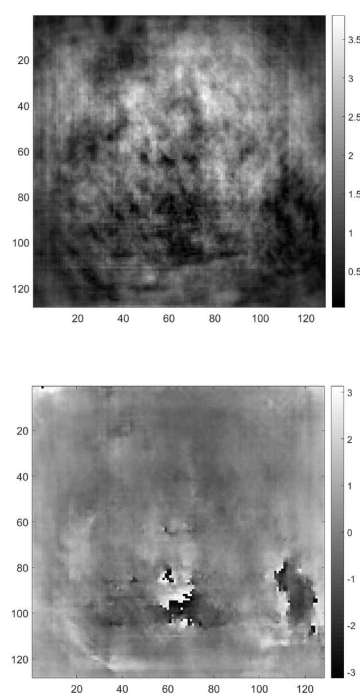

(b)
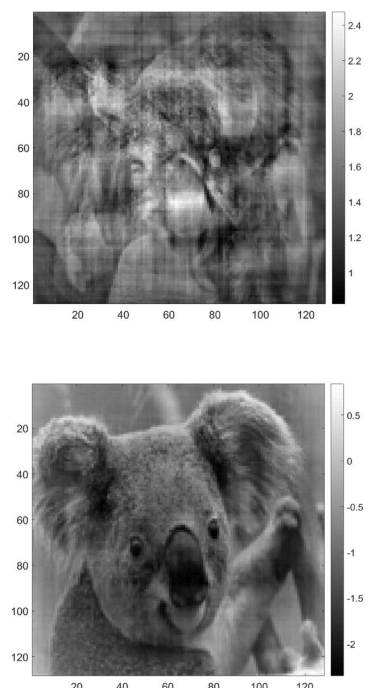

the sample are the same as the double-slit used above $(2.7 \mathrm{~m}$, $5.4 \mathrm{~m}$ and $10.8 \mathrm{~m}$ ). To satisfy the oversampling condition of CDI, the diffraction patterns are $512 \times 512$ grids. The diffraction pattern of the sample at $2.7 \mathrm{~m}$ is shown in Fig. 5(a). The profiles along the horizontal direction are taken to compare the contrast of diffraction pattern. As shown in Fig. 5(b), with the decrease of coherence, the contrast decreases because of the convolution effect of partial coherence. The complex sample is reconstructed using the Hybrid Input-Output algorithm (HIO) and Error Reduction method (ER) (Fienup, 1982) for each diffraction pattern, as shown in Fig. 6 . The following briefly shows how the reconstruction algorithm is applied (Fienup, 1982). First, a random phase is used to create the primary complexvalued distribution of the scattered wavefront $(F)$. Then, Fourier transformation is applied to create the complexvalued distribution of the object $(G)$. The generated $G$ is modulated by the support of object.

For the ER algorithm, 
(a)

$2.7 \mathrm{~m}$

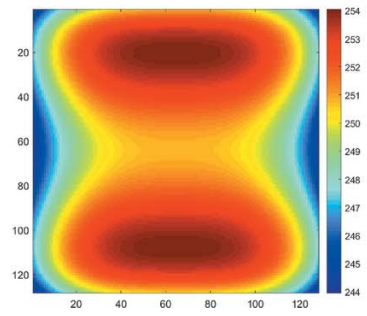

(b)

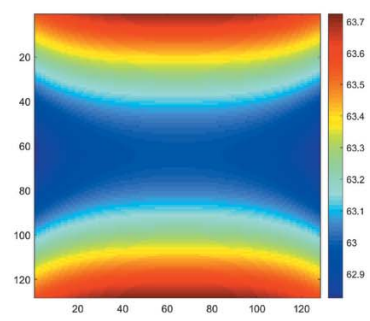

(c)

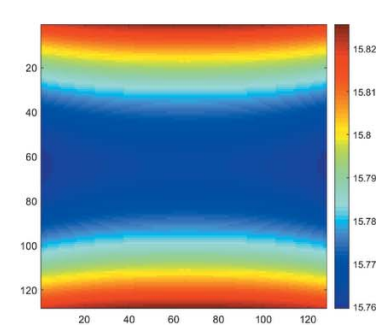

Figure 7

The intensity of the incident X-ray at the sample plane at the location of (a) $2.7 \mathrm{~m},(b) 5.4 \mathrm{~m}$ and (c) $10.8 \mathrm{~m}$.

$$
G_{k+1}(x, y)= \begin{cases}G_{k}(x, y), & \text { if }(x, y) \in \gamma \\ 0, & \text { if }(x, y) \notin \gamma\end{cases}
$$

For the HIO algorithm,

$$
G_{k+1}(x, y)= \begin{cases}G_{k}(x, y), & \text { if }(x, y) \in \gamma, \\ G_{k}^{\prime}(x, y)-\beta G_{k}(x, y), & \text { if }(x, y) \notin \gamma,\end{cases}
$$

where $G^{\prime}$ is the former generated complex-valued distribution of the object and $\beta$ is a constant.

From the reconstruction results, in order to obtain good reconstruction quality, the visibility of the interference fringes of any two points in the horizontal and vertical directions of the incident light field at the sample may be required to be greater than around 0.95 . However, as revealed above, the SNR will limit the resolution of the imaging result. Therefore, the total coherent flux and coherence should be balanced for beamline design. As shown in Fig. 7, the intensity of the incident X-ray at the sample plane is also calculated, and the structure of the light field distribution is clearly revealed.

\section{Conclusion}

In this paper, a wave optics model is proposed to analyze the influence of partial coherence on CDI, which is based on the finite size of the source and the effect of optics on the light field. Software has been developed to perform this process using Python (alpha version; Zhou \& Han, 2020). Thus, compared with $S R W$, a semi-quantitative method for beamline simulation is provided to verify different layouts of the beamline. The Hard X-ray Coherent Scattering beamline of HEPS is taken as an example whose partial coherence is analyzed by the visibility of double-slit interference. Then the double-slit is replaced with a complex sample and the diffraction patterns corresponding to different partial coherence are calculated, whose contrast decrease as the coherence decreases with the distance between secondary source and sample. Finally, the structures are reconstructed by phase iteration algorithm. According to the reconstruction results, in order to obtain good image quality of the complex sample, the visibility of the interference fringes of any two points in the horizontal and vertical directions of the incident light field at the sample might be around 0.95 . At the distance of $10.8 \mathrm{~m}$, the coherent length is calculated as $0.44 L \lambda / S=88 \mu \mathrm{m}$, where $L$ the real beamline would be possible.

is the distance from the secondary source to the screen $(10.8 \mathrm{~m}), \lambda$ is the wavelength $\left(1 \times 10^{-10} \mathrm{~m}\right)$, and $S$ is the FWHM of the secondary source $(8.2 \mu \mathrm{m})$. Therefore, the visibility observed using the flux accepted in a one-coherent length aperture is about 0.5 ; if the visibility is more than 0.95 , only $1 / 19$ of coherent flux will be used. The model can easily add more optics (such as a white beam mirror, monochromator, Kirkpatrick-Baez mirror, etc.) and take into account the influence of errors. Partial coherence analysis on

\section{Acknowledgements}

This work was supported by High Energy Photon Source (HEPS), a major national science and technology infrastructure in China.

\section{References}

Advanced Photon Source (2017). Advanced Photon Source Upgrade Project Preliminary Design Report. Advanced Photon Source, Argonne, IL, USA.

Chapman, H. N. \& Nugent, K. A. (2010). Nat. Photon. 4, 833-839.

Chubar, O. \& Elleaume, P. (1998). Proceedings of the Sixth European Particle Accelerator Conference (EPAC98), 22-26 June 1998, Stockholm, Sweden, pp. 1177-1179.

Clark, J. N., Huang, X., Harder, R. \& Robinson, I. K. (2012). Nat. Commun. 3, 993.

Diaz, A., Malkova, B., Holler, M., Guizar-Sicairos, M., Lima, E., Panneels, V., Pigino, G., Bittermann, A., Wettstein, L., Tomizaki, T., Bunk, O., Schertler, G., Ishikawa, T., Wepf, R. \& Menzel, A. (2015). J. Struct. Biol. 192, 461-469.

Dierolf, M., Menzel, A., Thibault, P., Schneider, P., Kewish, C. M., Wepf, R., Bunk, O. \& Pfeiffer, F. (2010). Nature, 467, 436-439.

ESRF (2015). ESRF Upgrade Program Phase II and Technical Design Study. ESRF, Grenoble, France.

Fienup, J. R. (1982). Appl. Opt. 21, 2758-2769.

Holler, M., Guizar-Sicairos, M., Tsai, E. H., Dinapoli, R., Müller, E., Bunk, O., Raabe, J. \& Aeppli, G. (2017). Nature, 543, 402-406.

Huang, X., Miao, H., Steinbrener, J., Nelson, J., Shapiro, D., Stewart, A., Turner, J. \& Jacobsen, C. (2009). Opt. Express, 17, 13541-13553.

Jiang, H., Xu, R., Chen, C. C., Yang, W., Fan, J., Tao, X., Song, C., Kohmura, Y., Xiao, T., Wang, Y., Fei, Y., Ishikawa, T., Mao, W. L. \& Miao, J. (2013). Phys. Rev. Lett. 110, 205501.

Kim, Y., Kim, C., Kwon, O. Y., Nam, D., Kim, S. S., Park, J. H., Kim, S., Gallagher-Jones, M., Kohmura, Y., Ishikawa, T., Song, C., Tae, G. \& Noh, D. Y. (2017). Sci. Rep. 7, 1850.

Klementiev, K. \& Chernikov, R. (2014). Proc. SPIE, 9209, 92090A.

Meng, X., Shi, X., Wang, Y., Reininger, R., Assoufid, L. \& Tai, R. (2017). J. Synchrotron Rad. 24, 954-962.

Miao, J., Charalambous, P., Kirz, J. \& Sayre, D. (1999). Nature, 400, 342-344.

Miao, J., Chen, C. C., Song, C., Nishino, Y., Kohmura, Y., Ishikawa, T., Ramunno-Johnson, D., Lee, T. \& Risbud, S. H. (2006). Phys. Rev. Lett. 97, 215503.

Miao, J., Ishikawa, T., Robinson, I. K. \& Murnane, M. M. (2015). Science, 348, 530-535.

Neutze, R., Wouts, R., van der Spoel, D., Weckert, E. \& Hajdu, J. (2000). Nature, 406, 752-757.

Nugent, K. A. (2010). Adv. Phys. 59, 1-99. 
Pfeifer, M. A., Williams, G. J., Vartanyants, I. A., Harder, R. \& Robinson, I. K. (2006). Nature, 442, 63-66.

Shen, Q., Bazarov, I. \& Thibault, P. (2004). J. Synchrotron Rad. 11, 432-438.

Song, C., Takagi, M., Park, J., Xu, R., Gallagher-Jones, M., Imamoto, N. \& Ishikawa, T. (2014). Biophys. J. 107, 1074-1081.

Ulvestad, A., Singer, A., Clark, J. N., Cho, H. M., Kim, J. W., Harder, R., Maser, J., Meng, Y. S. \& Shpyrko, O. G. (2015). Science, 348, 1344-1347.
Whitehead, L. W., Williams, G. J., Quiney, H. M., Vine, D. J., Dilanian, R. A., Flewett, S., Nugent, K. A., Peele, A. G., Balaur, E. \& McNulty, I. (2009). Phys. Rev. Lett. 103, 243902.

Williams, G. J., Quiney, H. M., Peele, A. G. \& Nugent, K. A. (2007). Phys. Rev. B, 75, 104102.

Yau, A., Cha, W., Kanan, M. W., Stephenson, G. B. \& Ulvestad, A. (2017). Science, 356, 739-742.

Zhou, L. \& Han, X. (2020). B4PC, https://github.com/Khan-Xu/ B4PC. 